\title{
Supplementary information: Kinetics of Photocontrollable Micelles: light-induced self-assembly and disassembly of azobenzene-based surfactants revealed by TR-SAXS
}

Reidar Lund ${ }^{1} *$, Geoffrey Brun ${ }^{2}$, Eloïse Chevallier ${ }^{2}$ Theyencheri Narayanan $^{3}$ and Christophe Tribet ${ }^{2}$

${ }^{1}$ Department of Chemistry, University of Oslo, P.O.Box 1033, Blindern, N-0315 Oslo, Norway.

${ }^{2}$ Ecole Normale Supérieure-PSL Research University, Département de Chimie, Sorbonne Universités UPMC Univ Paris 06, CNRS UMR 8640 PASTEUR, 24 rue Lhomond, 75005 Paris, France.

${ }^{3}$ European Synchrotron Radiation Facility (ESRF), 71 Avenue des Martyrs, F-38000 Grenoble, France.

*Email: reidar.lund@kjemi.uio.no
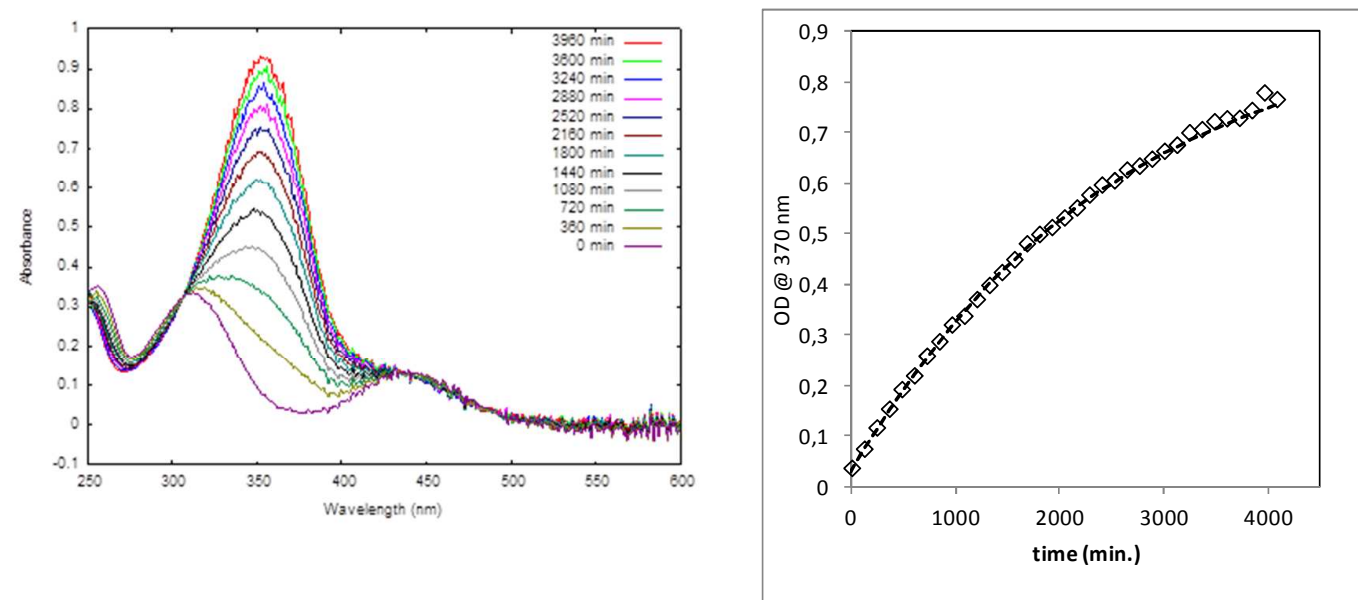

Figure S1. Thermal relaxation of a $17 \mathrm{mg} / \mathrm{L}$ AzoTMA in water:DMF (17 v/v\%). The solution was pre-exposed to UV light $(365 \mathrm{~nm}$ ) for $30 \mathrm{~min}$, and light was switched off at time zero. (A) UV-Vis absorption spectra recorded every 360 minutes; (B) plot of absorbance at $370 \mathrm{~nm}$ vs relaxation time and fit to the equation $O D(t)=A_{0}-B e^{-\frac{t}{\tau}}$, where $A_{0}=0.9 \pm 0.05$ is the DO measured prior to exposure to UV light (all trans state), B and $\square$ are adjustable parameters (here $\tau=2500$ minutes and $\left.\mathrm{B}=A_{0}-0.03\right)$. 


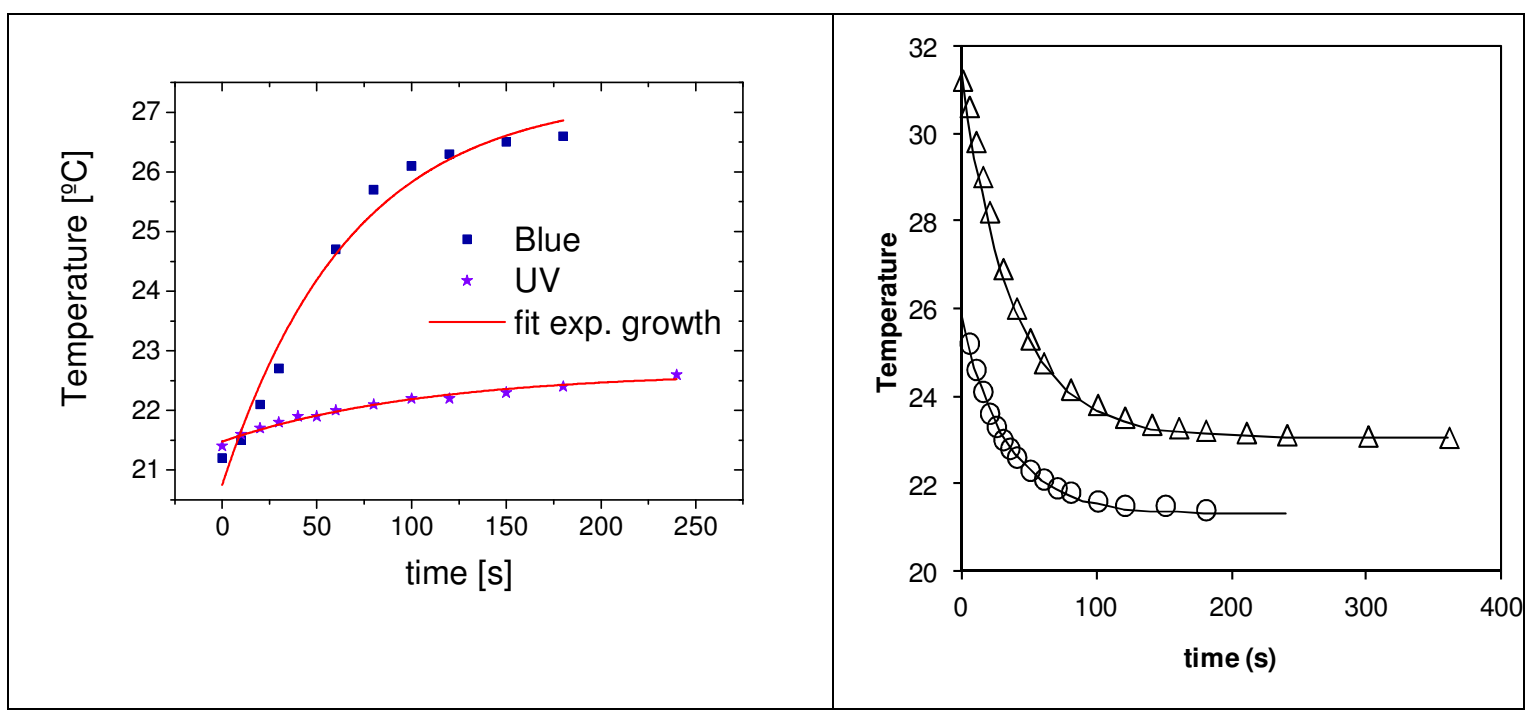

Figure S2. Temperature measured in SAXS capillary filled with a $10 \mathrm{~g} / \mathrm{L}$ AzoTMA solution in water:DMF 30\% and (A) exposed to UV or blue light at the same intensity as used in SAXS ; (B) the temperature after keeping the solution in the dark after exposure to UV (circles)land blue (triangles) light. Lines are fits to single exponential decay with a decay time of $36 \mathrm{~s}$. 


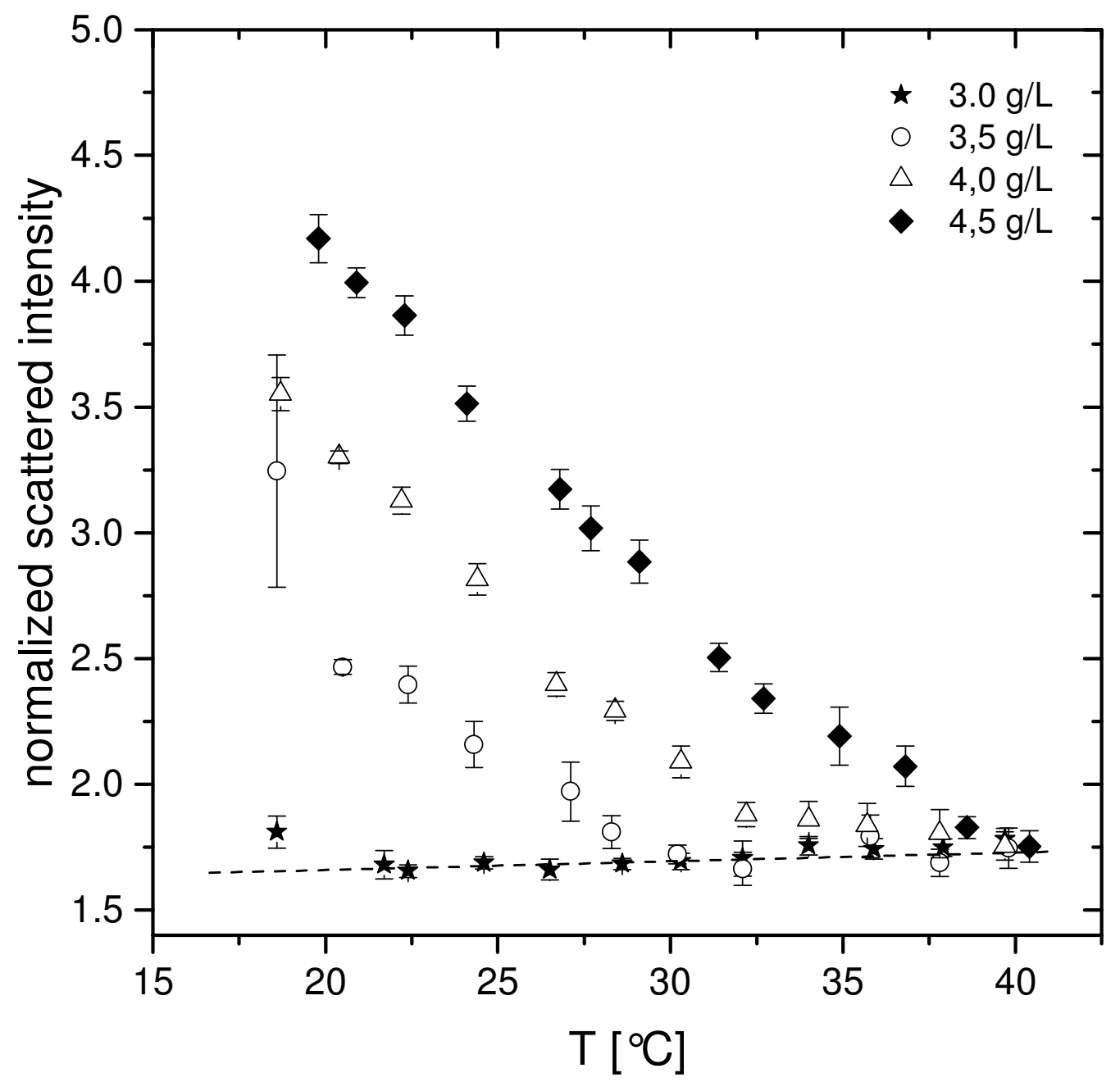

Figure S3. The scattered light intensity as a function of temperature for various concentrations of AzoTMA at 


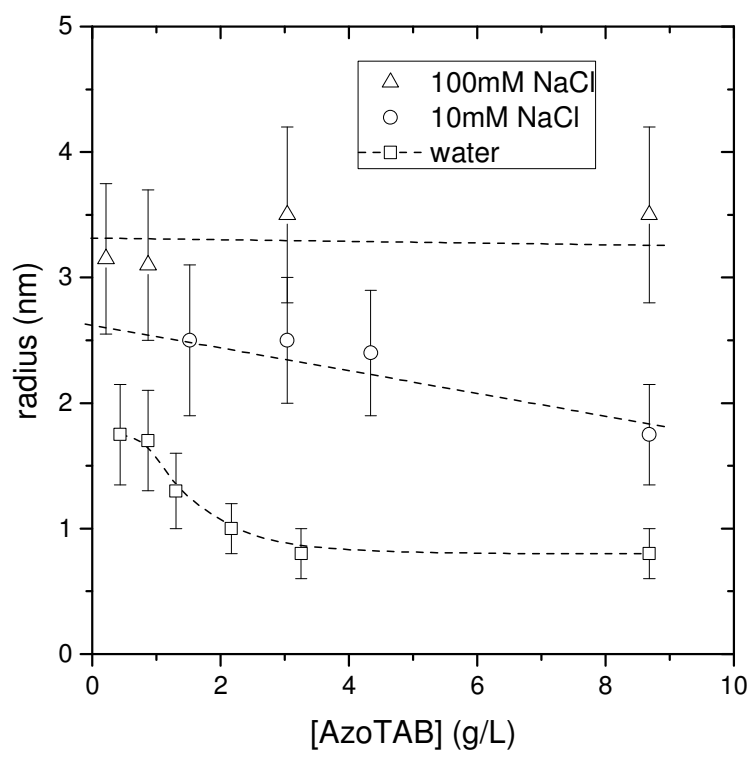

Figure S4. Radius of AzoTMA micelles as determined by dynamic light scattering at $150^{\circ}$ angle and $25^{\circ} \mathrm{C}$. in water $+\mathrm{NaCl}$, apparent radius calculated from the second order cumulant analysis. Lines are guides for the eye.

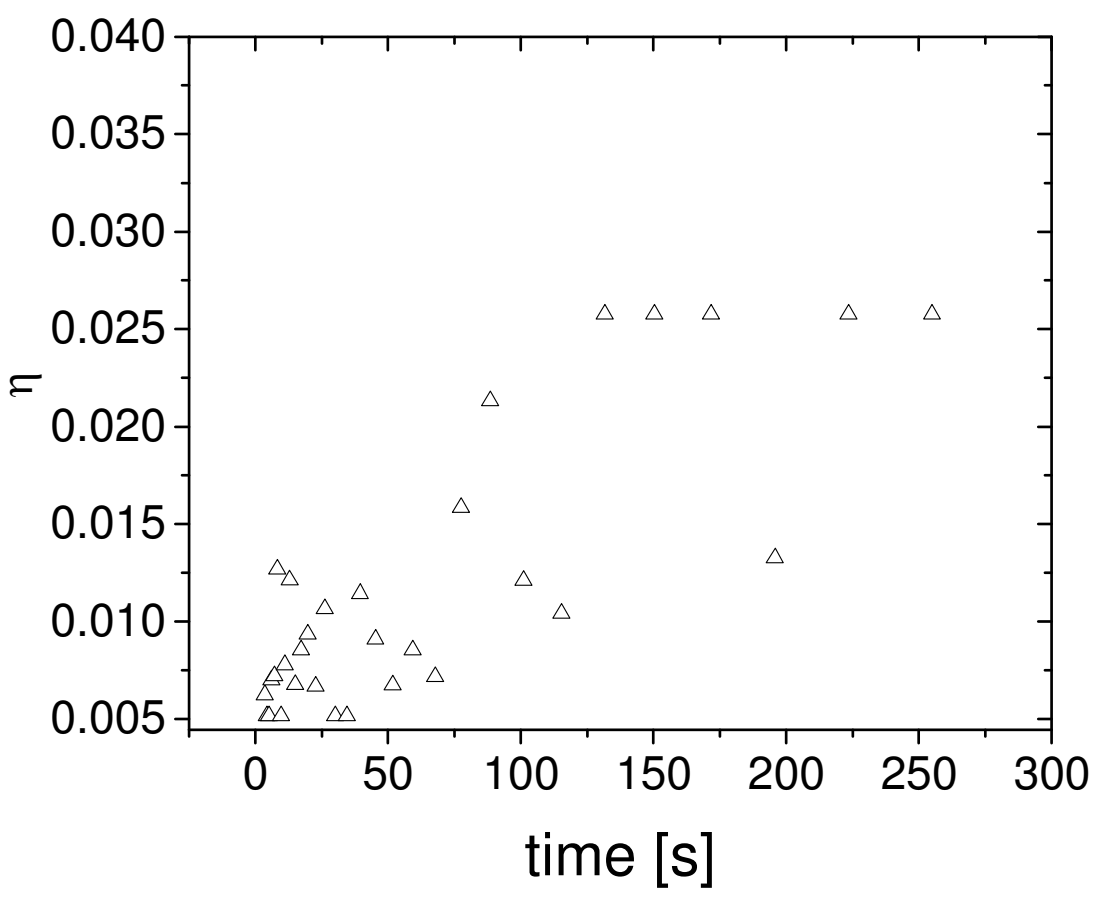

Figure S5. The effective volume fraction determined from the structure factor, $S(Q)$, describing the inter-particle interactions as a function of time for the $6 \mathrm{~g} / \mathrm{L}$ AzoTMA in 17\% DMF. Note that at large time, the fit parameters are increasingly unreliable due to the low fraction of micelles present. 


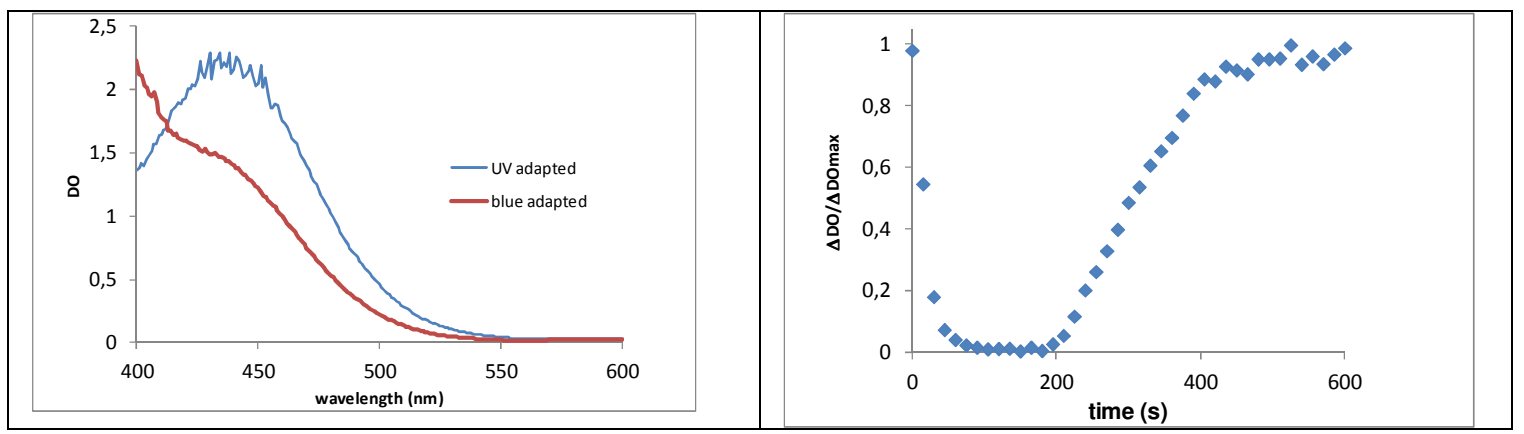

Figure S6. Variation upon exposure to light of the absorbance spectra of $5 \mathrm{~g} / \mathrm{L}$ azoTMA in water:DMF 17\% loaded quartz cell with in a $1 \mathrm{~mm}$ path length (left) spectra of solution exposed for 15 minutes ; (right) time variation upon exposure of UV-adapted solution to blue light $(11 \mathrm{~mW} / \mathrm{cm} 2)$ at time zero, then UV light $(2 \mathrm{~mW})$ at time $200 \mathrm{~s}$. with OD measured at $430 \mathrm{~nm}$ and $\Delta \mathrm{OD} / \Delta \mathrm{ODmax}={ }^{\prime}$ OD (t) - OD min ) / (OD max - OD min) 